\title{
Combined Lateral Calcaneal Lengthening Osteotomy and Dynamic Soft Tissue Reconstruction of Medial Foot in Adolescent Flexible Flatfoot
}

\author{
Mohamed Abd-El Aziz Mohamed Ali MD ${ }^{1 *}$, Mohammed Mansour MD ${ }^{2}$, Nagy Fouda MD $^{3}$ \\ ${ }^{1,2}$ Assisstant Professors of Orthopedic Surgery, Zagazig University, Egypt \\ ${ }^{3}$ Lecturer of Orthopedic Surgery, Zagazig University, Egypt
}

*Corresponding Author: Mohamed Abd-El Aziz Mohamed Ali MD, Assisstant Professor of Orthopedic Surgery, Zagazig University, Egypt.

\begin{abstract}
The objective of this study was to evaluate the operative management of flexible flatfoot in adolescent by calcaneal lengthening osteotomy described by Evans and dynamic soft tissue reconstruction of medial foot. To elevate the collapse of the medial longitudinal arch on the weight bearing state and correct the deformity which has three components heel valgus, forefoot abduction in addition to collapse of the medial longitudinal arch and centralizes the motion of the subtalar joint.
\end{abstract}

Methods: In our study, (42) feet in (21) patients who had symptomatic flatfoot were treated with lateral calcaneal lengthening (LCL) osteotomy, accessory navicular excision, anatomical reconstruction of the spring ligament complex (internal brace) augmentation and flexor digitorum longus (FDL) transfer to tibialis posterior tendon (TPT) and gastrocnemius recession. After LCL medial longitudinal arch is reestablished and soft tissues of medial foot usually require reconstruction. Thus, we decided to perform combination surgeries, we believe that using (FDL), as reported in this study, is a more anatomically correct procedure, supporting (TPT) without harmful effect on foot function, while simultaneously checking spring ligament.

Conclusion: It was observed that the preoperative severity of any of the components of the deformity did not affect significantly the final results. Pain and activity reduction and overall functions and appearance of the foot were improved significantly at the final outcome compared to the preoperative data.

All radiological measurements had a statistically significant improvement at the final follow up compared to the preoperative data.

In our study proved to be a good procedure to the surgical correction of symptomatic flexible flat foot as it is easy, corrects all the components of the deformity in one sitting, and can be done safely in children and adolescents.

Keywords: calcaneal, osteotomy, dynamic, adolescent, flatfoot

\section{INTRODUCTION}

Flatfoot is a complex multiplanar deformity both in children and adolescent. A clear definition of flatfoot is lacking and the limit between a physiological flatfoot (looked as normal) and a pathological flatfoot (required to be treated) is still uncertain. At present, foot kinematics in symptomatic and pathological flatfeet do not differ significantly, so pain may depend on tissue wear and subjective pain thresholds. The current trend is towards treatment of the deformity if it is painful and limiting activity, to relief from symptoms by restoring foot balance and alignment. (1)
Flatfoot is a common deformity manifested by medial rotation and plantar flexion of the talus, eversion of the calcaneus, collapsed medial arch and abduction of the forefoot. The bones of the foot are attached by fibrous structures, intrinsic muscles and extrinsic muscles. The important multiple joints of the foot are the ankle-joint, subtalar joint, transverse tarsal and tars metatarsal - (1).

The tendon of the (FDL) passes posterior to the distal end of the tibia and passes a shallow groove on the posterior surface of the talus, which is continuous with the groove on the plantar surface of the talar shelf. The tendon 
then crosses superficial the tendon of the flexor hullucis longus in the sole of the foot and flexor hallucis longus and (FDL) are an important role in maintaining the medial longitudinal arch in the foot. (2)
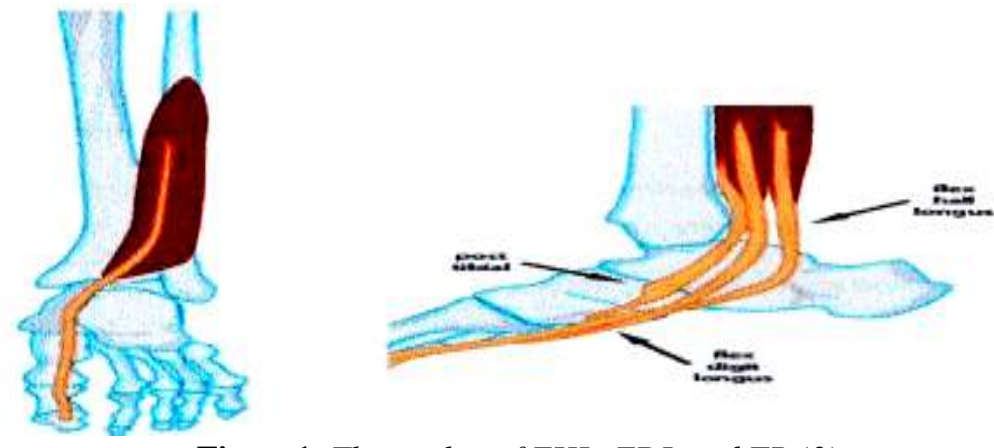

Figure1. The tendon of FHL, FDL and TP (3)

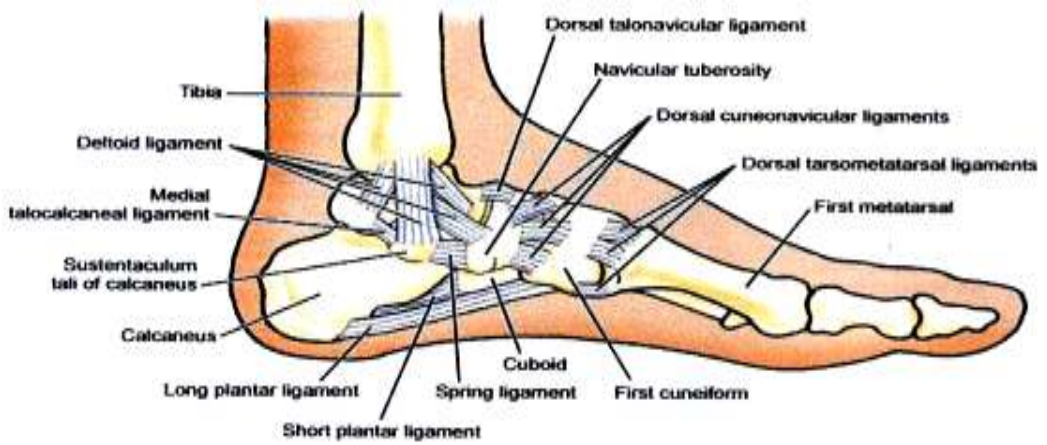

Figure2. Ligaments on the plantar surface of the foot (3)

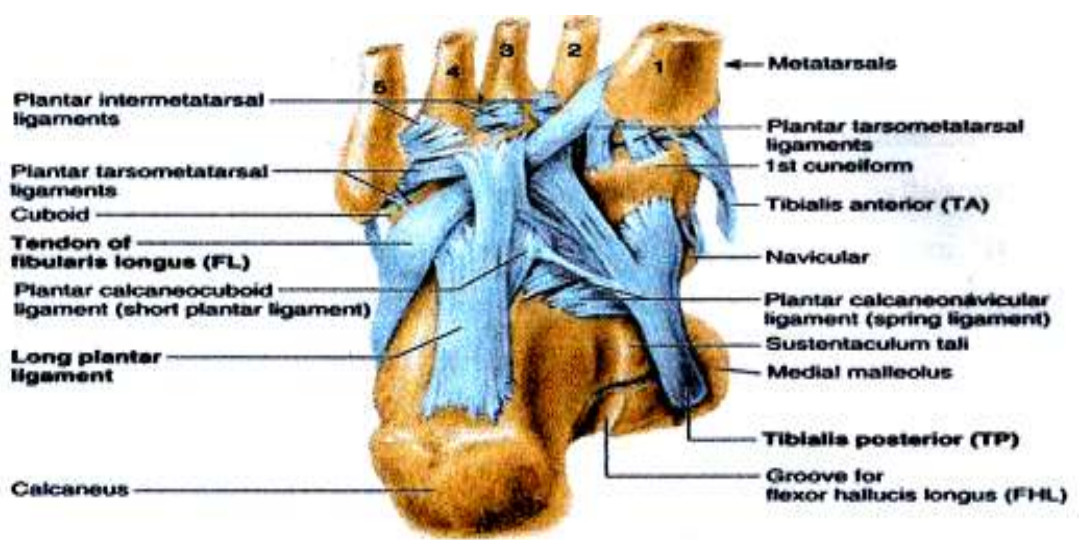

Figure3. Ligaments on the plantar surface of the foot (3)

The transverse tarsal articulations are the talonavicular and calcaneocuboid joints. These joints have parallel axes of rotation if the calcaneus is everted. If inverted, this will result in a non parallel axes. When parallel, the joint rotate in the same direction and the transverse tarsal articulation is flexible. When the axes are non- parallel, the articulation is rigid. (4)

\subsection{Clinical Features}

A-Symptoms: Foot and leg pain especially during standing and walking, difficulty in walking or running and easy fatigability in physical activities, symptoms increase as age advances and become marked when adolescence is reached. (9)

B- On examination. 1) Collapse of the medial longitudinal arch:

Grade 1 (mild), when the arch is slightly depressed.

Grade 2 (moderate), when the arch is absent but the medial border of the foot is straight.

Grade 3 (severe), when there is no arch and the medial border of the foot is convex. (9)

2) Heel valgus: grades depending on the measurement of the PTC angle, Normal $0^{\circ}$ to $5^{\circ}$, 
Mild $5^{\circ}$ to $10^{\circ}$, moderate $10^{\circ}$ to $15^{\circ}$, Severe > $15^{\circ}$. (10)

3) Positive toe extension test of jack:

4) Forefoot abduction: "too- many-toes" sign.

5) Prominence of the talar head on the medial side of the foot with subluxated talar head.
6) Pronated foot is a combination of downward and inward rotation of the midtarsal, abduction and eversion of the forefoot. (11)

7) Tightness of Achilles tendon is defined by limited ankle joint dorsiflexion. (12)

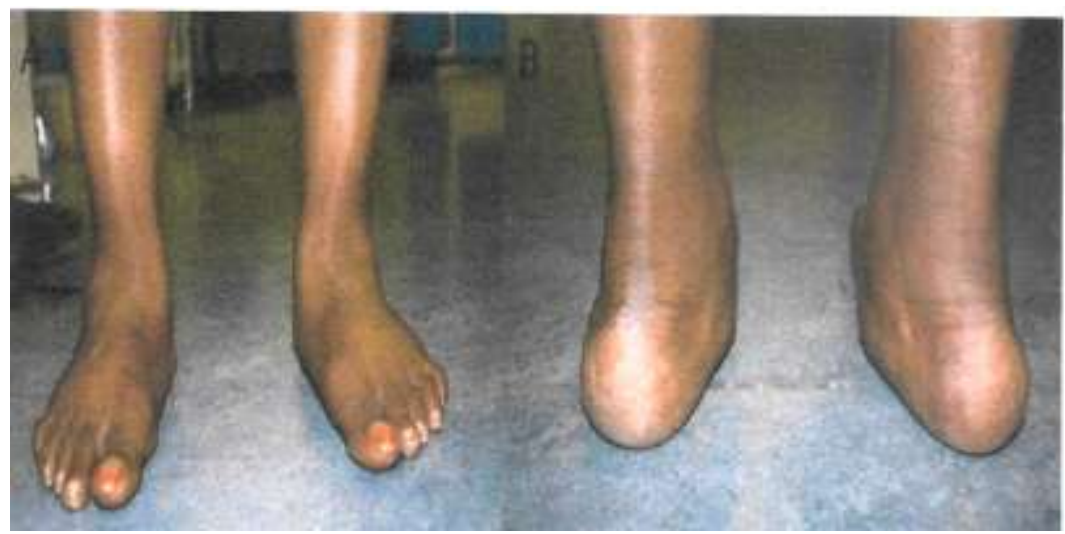

Figure4. Flexible flatfeet. A) Convex medial border with midfoot sag. Valgus hindfoot (4)
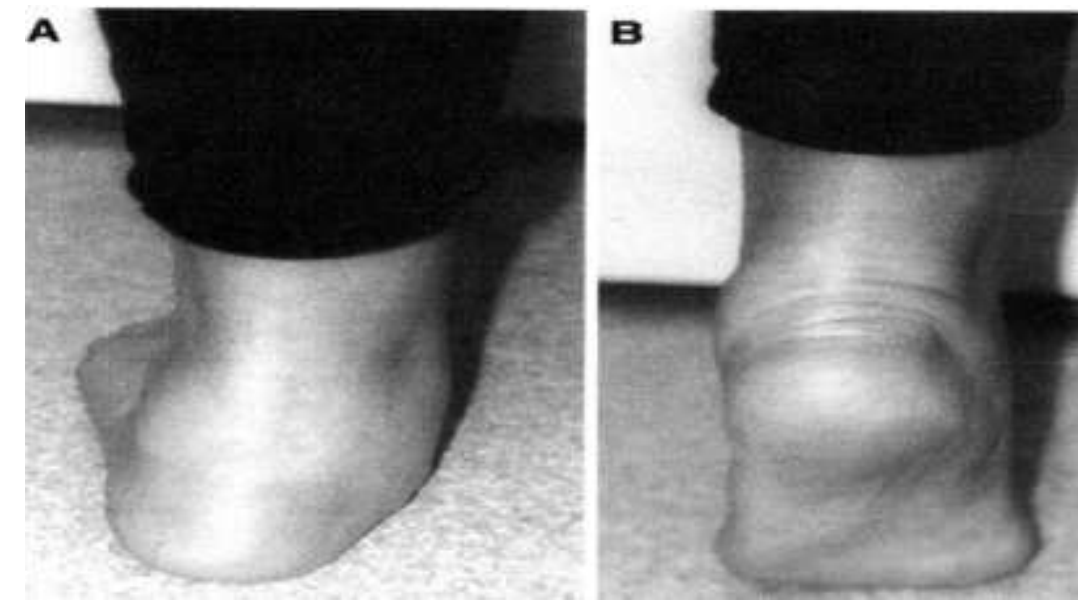

Figure5. (A) Weight-bearing left flexible flatfoot (B) In toe-standing, heel valgus converts to varus and the longitudinal arch can be seen (4)

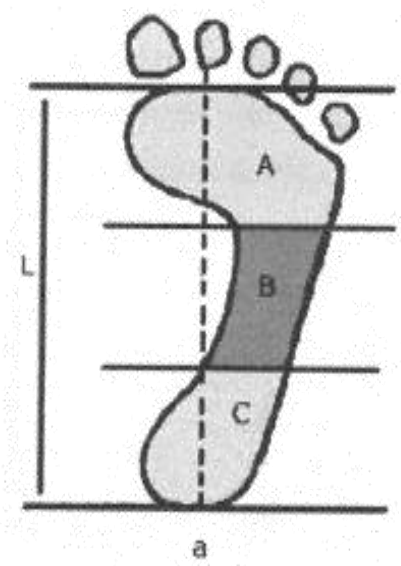

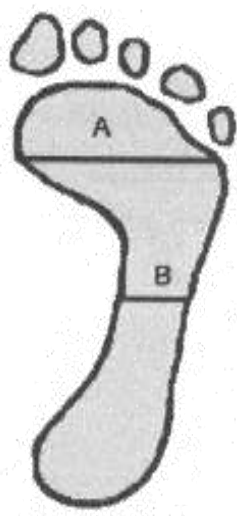

b

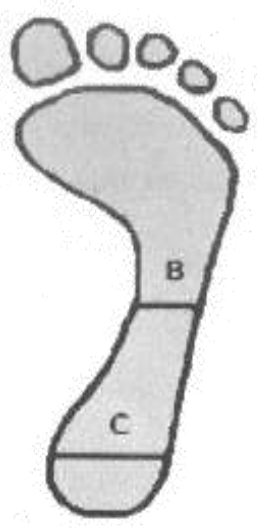

c

Figure6. (pedgraphy analysis, a) Arch index, A-forefoof, B-midfoot, $C$-hindfoot, L length of the foot. Arch index $=B / A+B+C ; b)$ Chippaux-Smirak index B/A $x 100 \% c$ ) Staheli arch index $=B / C \times 100 \%$ (5) 

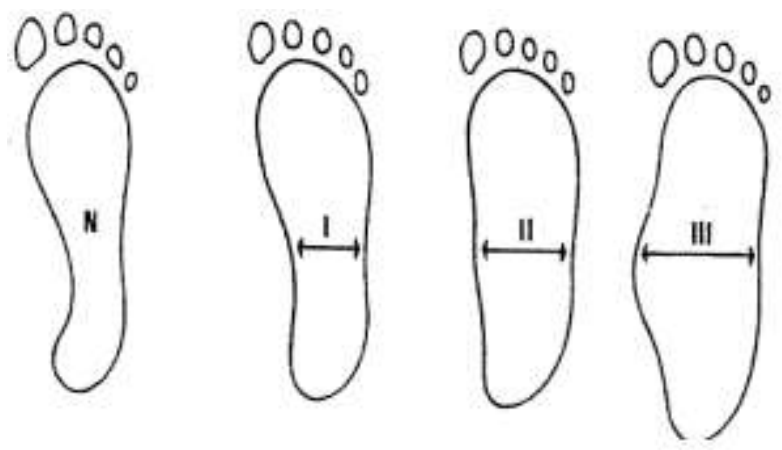

\section{$\mathrm{N}=$ Normal \\ $1=$ Grade 1 \\ $2=$ Grade 2 \\ $3=$ Grade 3}

Figure7. (18) The plantar footprint (Denis) was graded into three grades of flatfoot:, the support of the lateral edge of the foot is half of that of the metatarsal support grade 1; , the support of the central zone and forefoot are equal grade 2; and the support in the central zone of the foot is greater than the width of the metatarsal support grade 3. (6)

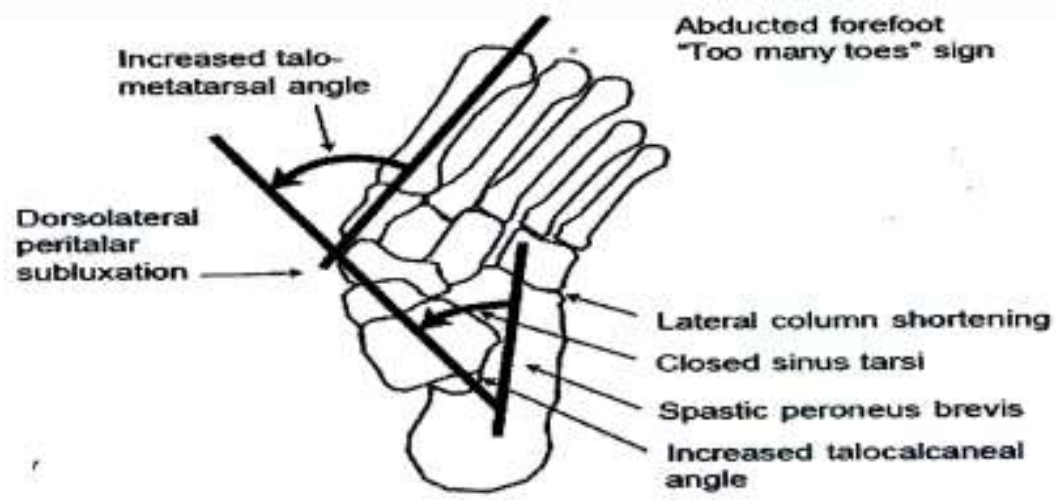

Figure8. Weight bearing AP in flat foot show increase talometatarsal angle (forefoot abduction) and increase talocalcaneal angIe. (7)

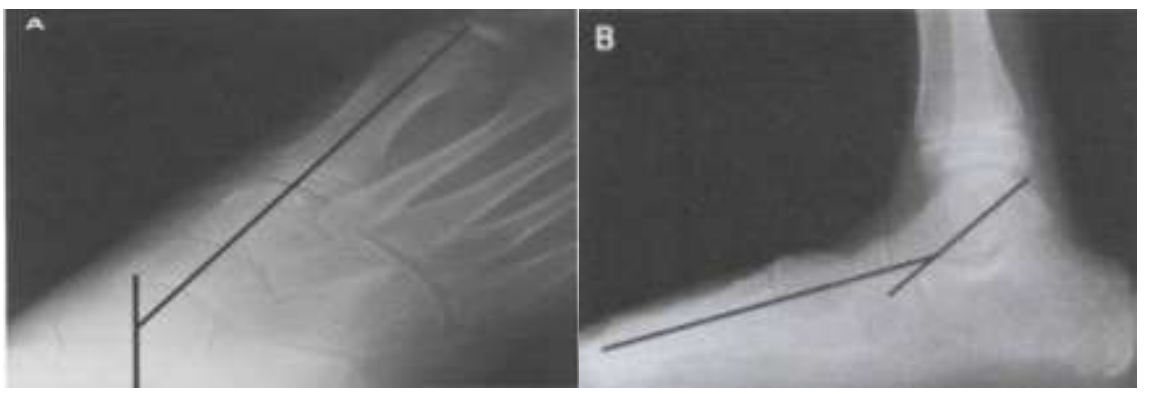

Figure9. (A) Standing radiographs of a flatfoot demonstrating talus and first metatarsal axis lines crossing at the center of rotation of angulation (CORA) in the center of the head of the talus, indicating a single deformity at the talo-navicular joint, A) Anteropostenor view. (B) Lateral view. (7)

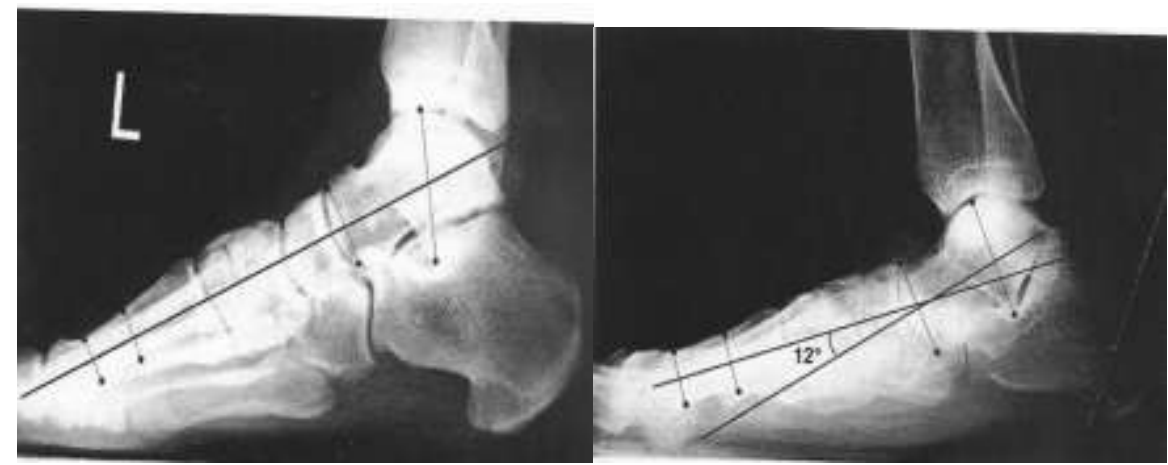

Figure10. (A) Normal Meary's angle. The long axis of the talus is in line with that of the first metatarsal. B) The long axis of the talus is directed plantar ward in relation to the first metatarsal, consistent with flatfoot. Lateral talar - 1 St Metatarsal angle. (7) 


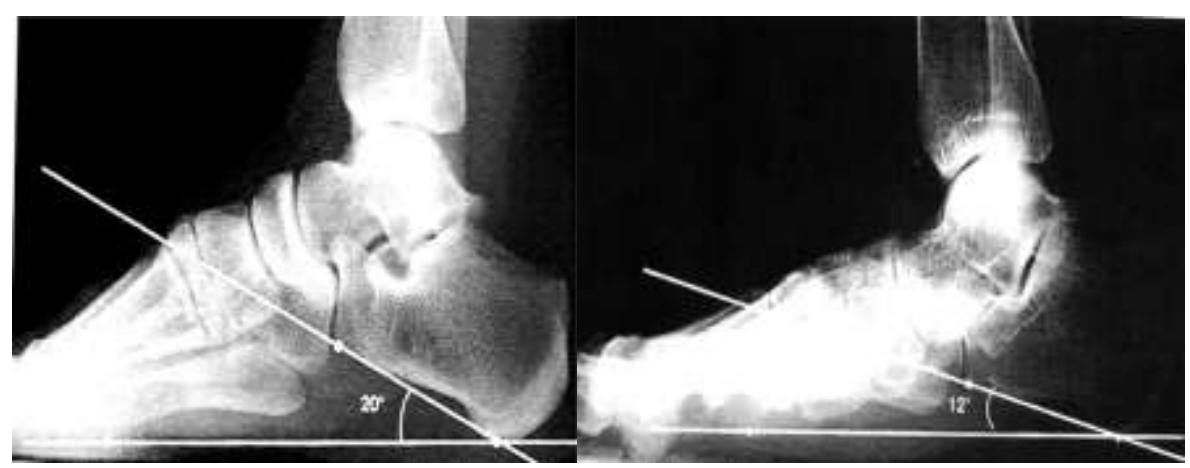

Figure11. A) Normal calcaneal pitch. B) Decreased calcaneal pitch in flatfoot (8)

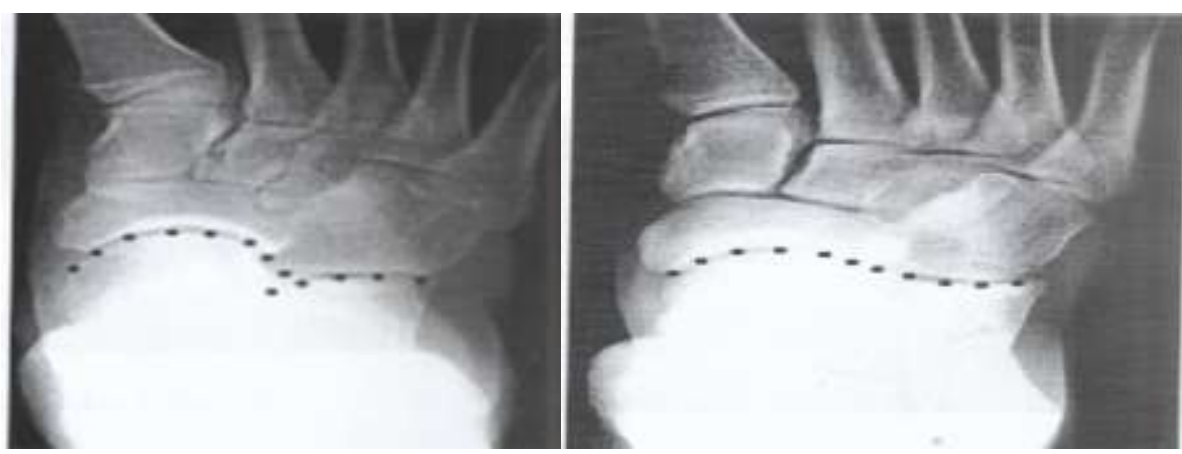

Figure12. A) Broken Cyma line of flatfoot. B) Normal AP cyma line: Line between talonavicular joint and calcaneocuboid joint is smooth and continuous. (8)

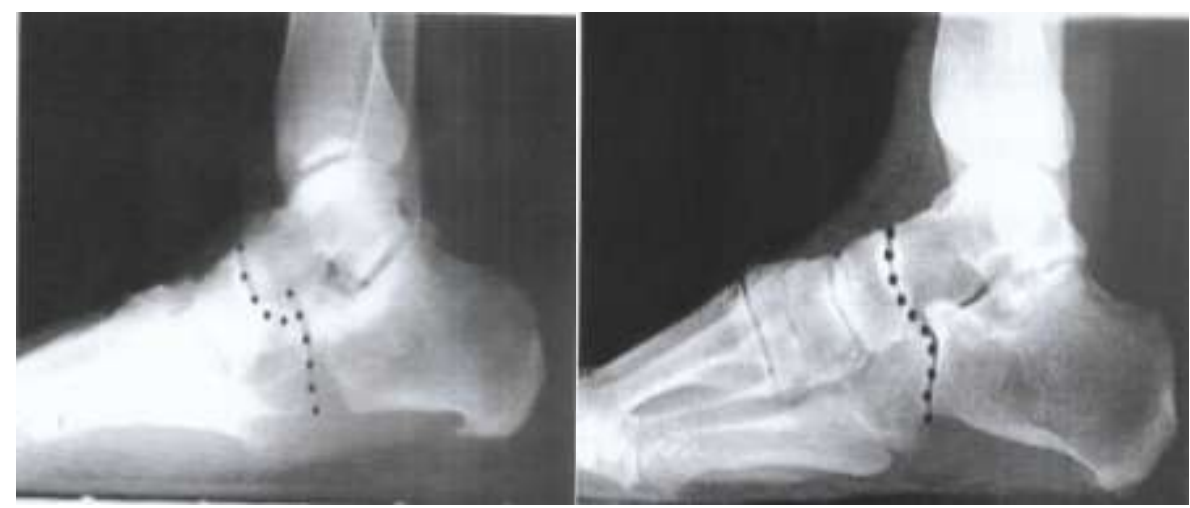

Figure13. A) Broken Cyma line of pesplanus. B) Line connecting talonavicular joint and calcaneocuboid joint is smooth and continuous. (8)

\subsection{Operative Procedure}

The patients who are shoing difficulty in daily routine activities because of pain and that have callosities and feet strain on the medial side of the foot, causing discomfort while walking, should be treated.

the calcaneal lengthening osteotomy was first described by Evans in 1975, and was provided as an alternative to triple arthrodesis in calcaneovalgus deformities owing to various etiologies. The technique was then popularized and modified by Mosca for treatment of FFF. This procedure clinically manages both the hindfoot and midfoot, and reconstructs the medial longitudinal arch. (13)
Evans considered that the lateral column of the flatfoot was shorter than the medial column. For painful flatfeet, he equalized the lengths of the columns by putting a corticocancellous graft into an osteotomy of the anterior calcaneus that was made $1.5 \mathrm{~cm}$ proximal to, and parallel with, the calcaneocuboid joint. By lengthening the calcaneus, the heel valgus, talo-navicular sag, and lateral subluxation of the navicular on the head of the talus could all be simultaneously reconstruted (13) In our study, caicaneal lengthening osteotomy is recommended for midtarsai and to some extent, mix category. On the other hand, calcaneal lengthening osteotomy is not advised in the subtalar category and is contraindicated in pes piano cavus (22). 
Our study reportet that calcaneal lengthening osteotomy is an appropriate technique for the correction of the idiopathic flexible flat foot and performing soft tissue procedures on these patients resulted in excellent results. Soft tissue reconstruction and in our study is aimed toward more anatomic spring ligament and posterior tibialis tendon reconstructions. Arthrodesis restricts range of motion leading to early degenerative changes in adjacent joints. (15)

\subsection{Surgical Technique}

Curvilinear incision was done distal to the sinus tarsi and 1 to $1.5 \mathrm{~cm}$ proximal to the calcaneocuboid joint, avoids the dorsal cutaneous nerves and provides access to the lateral wall of the anterior portion of the calcaneus, although care must be taken to protect the peroneal tendons and the sural nerve. (16)

The autogenous bone graft (fibular) wedge was sized to fit the corrected alignment. The graft was fashioned into a triangle, with its cortical base measuring 7 to $10 \mathrm{~mm}$ in width. The

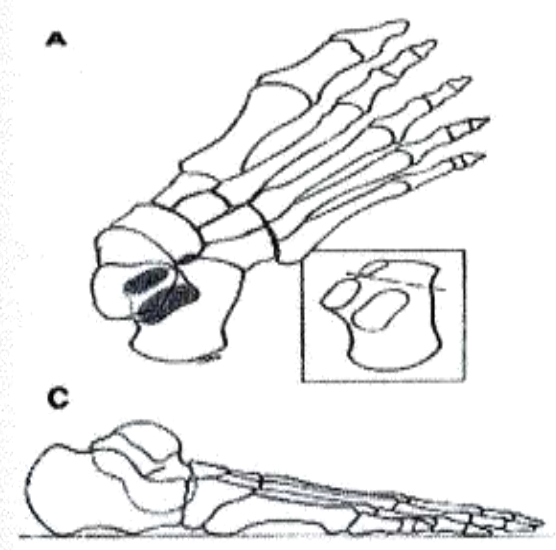

Figure14. Lateral calcaneal lengthening osteotomy (14)

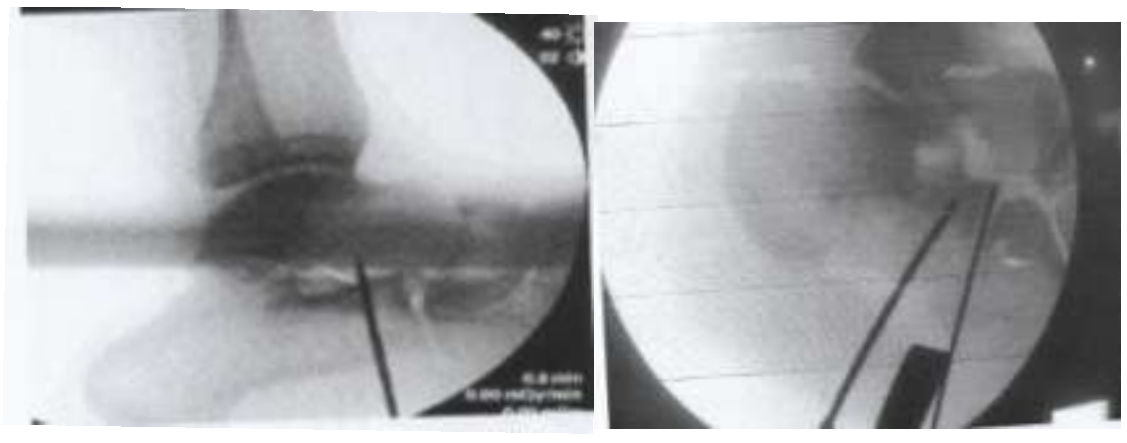

Figure15. C arm showing calcaniocuboid joint and site of osteotomy

FDL tendon was then transferred. It was placed at level distal to Henry's knot, as described by Haddad and Mann, and prepared tendon was done through hole in navicular bone and sutured

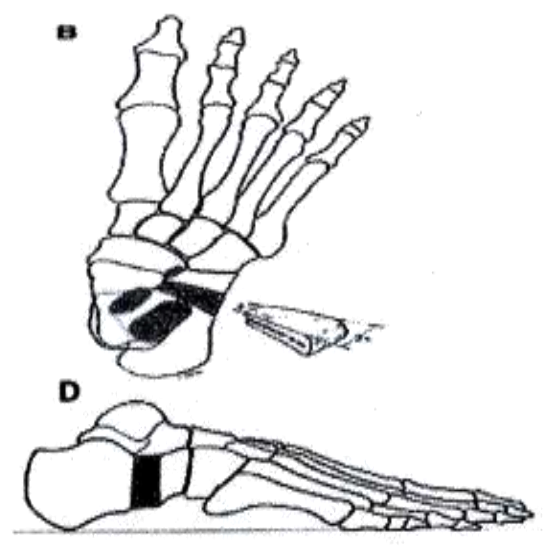

cortical wide base was oriented lateral and apex medial, and then attempted into its final position and the graft secured with two $\mathrm{k}$ wires for fixation. We fix the (fibular) grft and calceneocubiod joint to avoied subluxation of the joint. (16)

A medial skin indision was done over the course of the posterior tibial tendon from the malleolus to the plantar edge of the first metatarsal. The tendon sheath is opened, the posterior tibial tendon is exposed and attenuated portions, of the tendon are debrided. The medial talonavicular capsule is evaluted and the spring ligament is identified. the spring ligament, the ends were cleared and the remainder imbricated in a "pants-over-vest" fashion using multiple braided non-absorbable size sutures. If the capsule or spring ligament were redundant or attenuated, but not torn, a vertical incision is made in the capsule and imbrication is performed. The flexor digitorum Iongus tendon is defined and sutured side by side to the (TPT) or anch ored to the navicular bone. to itself under tension. If loose spring ligament was plicated with 2 non-absorbable, braided sutures and interal brace was done. 
After LCL medial longitudinal arch is re established and soft tissues of medial foot usually require stretch ing. Thus, we decided to perform combination surgeries, we believed that using FDL, as reported in this study, is a more

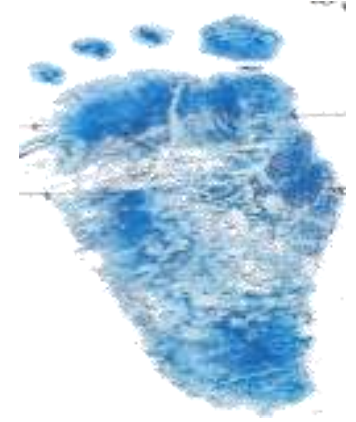

Figure16. Pedograph pre-operative and postoperative

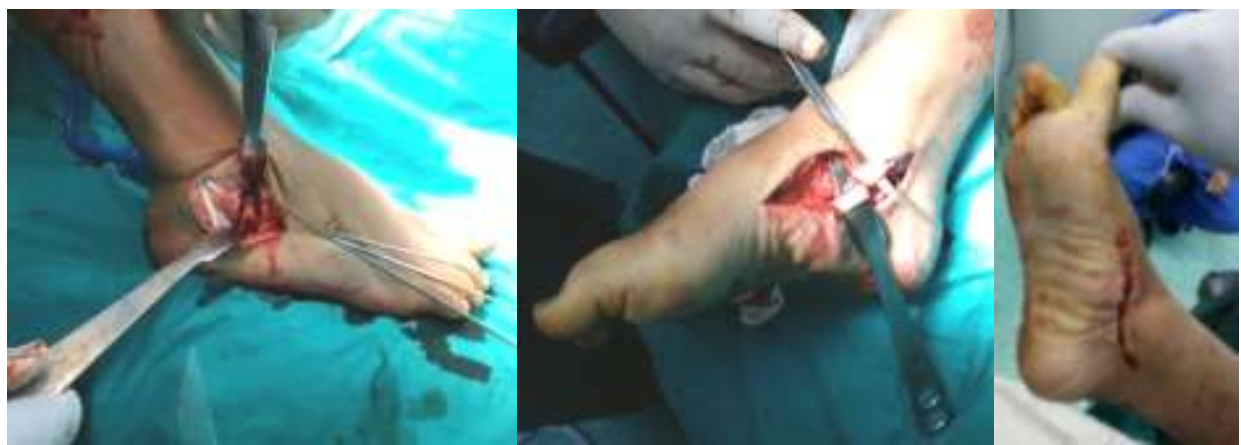

Figure17. The autogenous bone graft (fibular) wedge was sized to fit the corrected alignment FDL is passed from plantar to dorsal and talonavicular capsule and spring ligament sutures.

\section{MATERIALS AND MethodS}

Patients (42 feet) underwent lateral calacaneal lengthening combined with talonavicular capsule and spring ligament repair/reefing and flexor digi torum longus tendon transfer to the navicular or side to side to (TPT) for the treatment of flexible flatfoot deformities. The average age of the patients was 10 years (range 6-15), and (9) male and (8) female. Clinically, no fixed deformities of the subtalar or transverse tarsal joints were present in the study anatomically correct procedure, supporting (TPT) without harmful effect on foot function with simulta neously checking spring ligament.

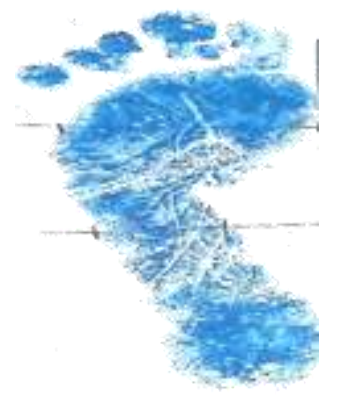

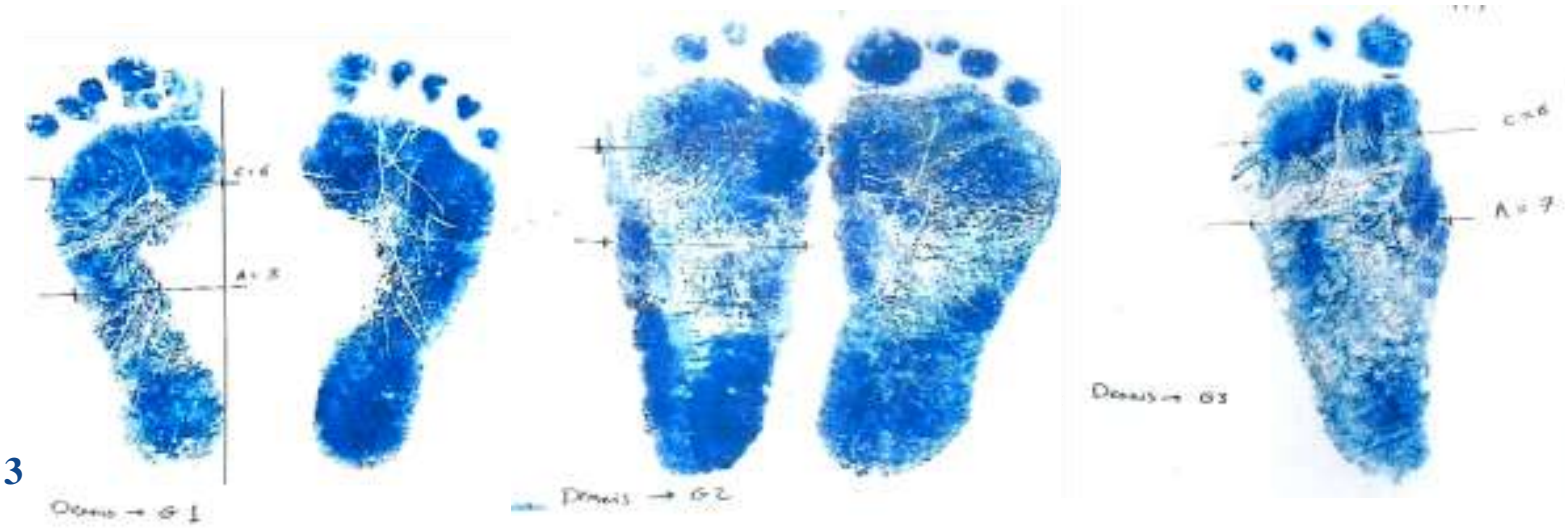

population. All patients had failed conserva tive management of their deformities. 36 of 42 feet $(88 \%)$ had prior treatment with custom mold ed closed cell foam foot orthoses. The average follow- up was 26 months (9-52). Average duration of symp toms prior to surgery was 18 months (range 6-48). Nine patients (10 feet) had lateral pain from subfibular impingement, and 2 patients were noted to have degen erative changes of the subtalar joint.

Fibular bone graft was used in 42 feet.

Figure18. Footprint classification of Denis 
Patient evaluation included detailed clinical and functional analysis including (AOFAS) foot rating score and detailed physical examination. (9).Average AOFAS foot score postoperatively was 86. A summary of demographics and perioperative information and clinical Outcomes achieved a total mean of 8 points (range, 5-10 points). 4 patients were not able to per forma single-leg raise.

The average AOFAS ankle-hindfoot score improved from 68 points (range, 52-87 points) preopera tively to 89 points (range, 63-100 points; $\mathrm{P}<.001)$ postoperatively. The AOFAS hal lux score at final follow-up averaged 84 points (range, 71-95 points). The combination of the flexor digitorum longus tendon transfer and spring ligament repair with calcaneal lengthening is an effective and reliable procedure which provides excellent correction of hindfoot valgus as well as forefoot abduction and restoration of the height of the longitudinal arch.

Result of pedographs of foot according to (Denis classification) preoperative was increased in midfoot contact area from $66 \%$ to $25 \%$ and it was statistically significant ( $\mathrm{p}$ value: $0.04 \%)$.

All patients were evaluated pre- and postoperatively with standard weight bearing radiographs.

\subsection{Case Presentation}
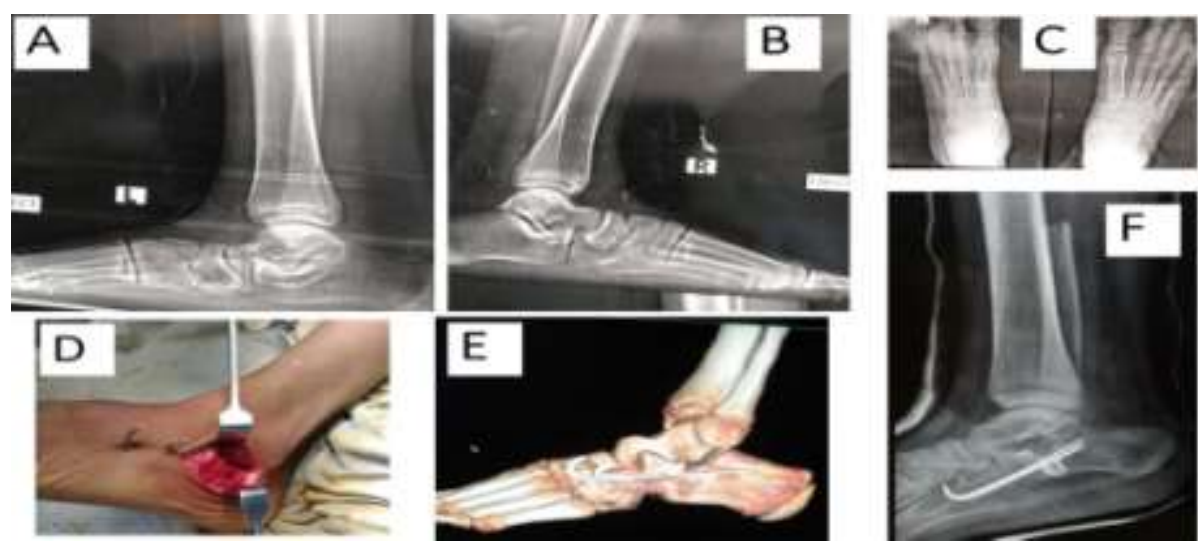

Case1. A, B, C) Preoperative-X Ray, D) Intraoperative Photo after correction, E, F) Postoperative CT, and Plain X Ray.

Radiographic results included pre- and postoperative determination of AP talo-lst metatarsal angle, talonavicular coverage angle (as described by Sangeorozan et ala, lateral talolst metatarsal angle, lateral talocalcaneal angle, and the distance from the plantar edge of the medial cuneiform to the floor on the lateral projection.

Standing radiographic analysis demonstrated an average improvement in the AP talo-1st metatarsal angle of $6^{\circ}\left(12.3^{\circ}\right.$ preoperative, $6.3^{\circ}$ postoperative).

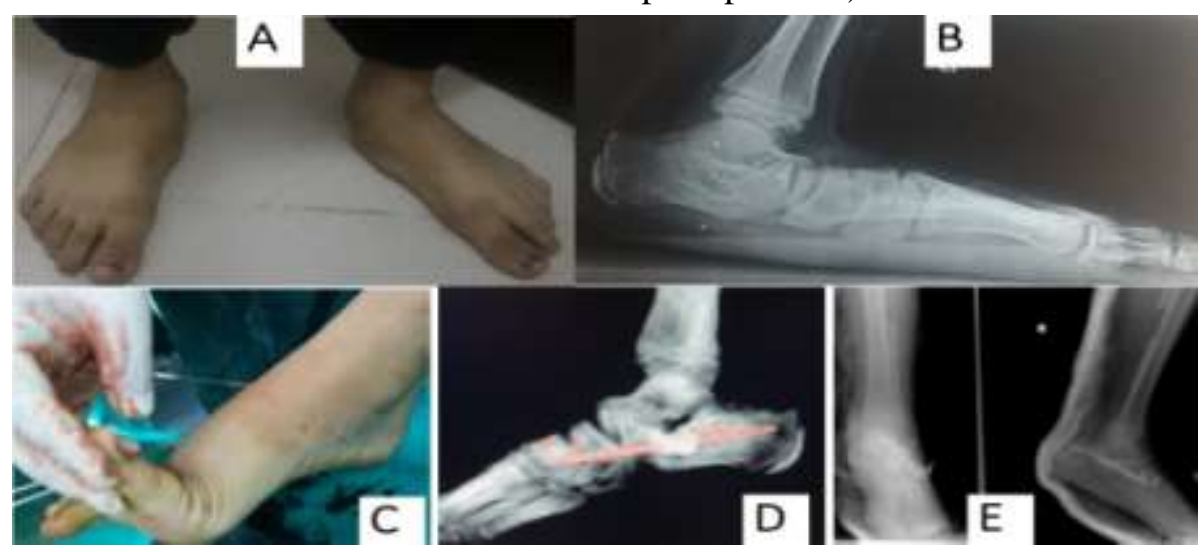

Case2. A) Preoperative photo with thumb elevation flexible flat foot, B) Preoperative X-ray lateral view, C) intraoperative photo after correction, D \& E) postoperative CT, and plain X-ray.

The talonavicular coverage angle improved an average of $20.4^{\circ}\left(36^{\circ}\right.$ preoperative, $15.6^{\circ}$ postoperative). The lateral talo-1st metatarsal angle improved an average of $9.2^{\circ}\left(12.7^{\circ}\right.$ preoperative, $3.5^{\circ}$ postoperative). The lateral talocalcaneal angle decreased an average of 6.1 
$\left(34.9^{\circ}\right.$ preoperative, $28.8^{\circ}$ postoperative). The distance of the medial cuneiform to the floor on the lateral radiograph averaged (12) $\mathrm{mm}$ preoperatively and (17) $\mathrm{mm}$ postoperatively (average improvement (5) $\mathrm{mm}$ ).

Table1. Preoperative and Postoperative Measurements in Radiography Results

\begin{tabular}{|l|l|l|l|l|}
\hline & & $\begin{array}{c}\text { mean preop value } \\
\text { (degrees) }\end{array}$ & $\begin{array}{c}\text { mean postop value } \\
\text { (degrees) }\end{array}$ & Palue \\
\hline lateral view & talo-first metatarsal angle & 12.7 & 3.5 & 0.002 \\
\hline & Calcaneal inclination angle & 13.9 & 22.5 & 0.005 \\
\hline & talo Calcaneal angle & 34.9 & 28.8 & 0.002 \\
\hline & talohorizontal angle & 28.5 & 18.2 & 0.002 \\
\hline AP view & distance of medial cuneiform to floor & 12 & 18 & 0.002 \\
\hline & talo-first metatarsal angle & 12.7 & 3.5 & 0.01 \\
\hline & talonavicular coverage angle & 36 & 15.6 & 0.007 \\
\hline & talo Calcaneal angle & 25.9 & 19.5 & 0.03 \\
\hline
\end{tabular}

p 0.05 was accepted as statistically significant, AP: Anteroposterior

\section{DISCUSSION}

In our results, there were significant improvements in AOFAS scores. Patient pain diminished or disappeared, and they achieved better walking distance. All patients and their families are satisfied with surgery. Results showed a significant change in the talar first metatarsal angle $(\mathrm{p}=0.02)$, talonavicular angle $(\mathrm{p}=0.04)$ and medial cuneiform height $(\mathrm{p}=$ $0.03)$ in lateral talohorizontal angle $(\mathrm{p}=0.002)$, confirming radiological indication of effectiveness of combined treatment.

In our cases, pedographic measurements showed a significant change in anterolateral pressure was found after the performance of the osteotomy and tendon transfer combined with anatomical reconstruction of the spring ligament complex (internal brace) augmentation ( $\mathrm{p}=$ 0.01). (LCL) osteotomy decreases pressure in lateral column of the foot. Reduction in pressure is thought to be source of reduced lateral column pain. We document preoperative or postoperative pedographic measurements, significant improvement.

Idiopathic flat foot can differ in magnitude amongst patients. Although asymptomatic in most, the severity of symptoms and fui disturbance is severe in a few cases and may not respond to conservative measures. These patients enter a vicious cycle of physical limitation due to foot pain, calf muscles strain, callosity, and ankle sprain as well as limitations in choosing a shoe. This restricted mobility results in obesity, which in turn exacerbates their symptoms $(14,15)$.

Martinus made a study in 112 feet in 102 patients were analysed (age, 57.6 (13-82), 42\% male). In 12 feet (9\%) wound healing delay without further surgical measures was registered. All patients made a full weight bearing during the 7 th postoperative week. Until followup, revision surgery was done in 3 patients (fusion calcaneocuboid joint $(n=2)$, correction triple arthrodesis .101 feet (90\%) completed 2-year- followup. TMT dorsoplantar/lateralfindex and VAS FA scores were marked increased, and posterior tibialis insufficiency stage, pedographic midfoot Contact area and force percentage were decreased (each $\mathrm{p}$ <.05). Assessment was performed before surgery and at 2-yearfollowup including clinical examination (with staging of posterior tibialis insufficiency) weight bearing radiographs (Talo-Ist metatarsal angles (TMT)), pedography (increased midfoot contact area and force) and Visual Analogue Scale Foot and Ankle (VAS FA).

Calcaneal lengthening osteotomy, first done by Evans in 1975 is one of the surgical techniques for the treatment of flat foot (17). In 1983, Phillips evaluated patients who were done by "Evans" technique. He reported satisfactory results at long term follow up (18). In 1995 Mosca modfied Evans' technique by doing a medial cuneiform osteotomy to achieve a more balanced correction (19).

Unlike most other previous studies, we reported the outcomes of surgical intervention on idiopathic flexible flat foot. In Mosca's cohort, 26 out of 31 flat feet were due to neuromuscular disorders (9). Dogan et al. found only 4 idiopathic flat feet in a total of 13 patients who underwent the calcaneal lengthening osteotomy. Although idiopathic flexible flat foot is the most common type, the efficacy of surgery on this group is yet to be robustly reported. (20)

Arthroereisis of the subtalar joint with synthetic implant may result foreign body reactions, 
infection, pain, incomplete deformity correction, and need for another surgery (21).

We believe a satisfactory result in this surgery relies on two factors: appropriate patient selection and surgical technique.

We recommend this surgery for patients with marked symptoms resulting in functional disturbance, who have failed to be treated by conservative treatments for 6 months. We don't advise this surgery for cosmetic indications. The need for the confirmation of the diagnosis cannot be over emphasized. In our study we do calcaneal lengthening osteotomy in midtarsal flat foot which is distinguishable from subtalar type by physical examination. The lateral column in midtarsal flat foot is short and there is a significant abduction in forefoot and "too many toe sign" is present. Furthermore, subtalar motion is relatively well preserved and hind foot valgus is not prominent. Standardized standing radiographies confirm the clinical diagnosis. Radiographs also help to find other pathologies. In the lateral radiograph, the first talometatarsal angle diagnoses a loss of the medial arch. Talonavicular and naviculocuneiform angles define where the medial arch collapse. In the midtarsal type the deformity is in the naviculocuneiform joint as reporated by the angle, whereas in the subtalar type the main deformity is from the talonavicular joint. The calcaneal inclination angle, is measured on the lateral radiograph. As a measure of hind equinus this index has been diagnosed by the lateral tibiotalar angle. If a normal or increased calcaneal inclination angle is present in a patient with flat foot, pes piano valgus is unlikely and pes piano cavus should be suspected. This is important to note as calcaneal lengthening osteotomy is contraindicated in this type.

Standing calcaneus-fifth metatarsal angle can be measured in the AP view. This angle helps us to choose the appropriate surgical technique and in measuring the correction following the osteotomy. it is more useful than talofirst metatarsal angle on the same pro jection. A negative calcaneus-fifth metatarsal angle demonstrates forefoot abduction and shortening of lateral column associated with the midtarsal type. After we were performing calcaneal lengthening osteotomy this angle becomes closer to zero. A positive angle becomes positive can indicate overcorrection. The AP talocalcaneal angle is a radiolographic sign for hind foot valgus. This angle in-creases significantly in flat foot patients with subtalar pathology. Our study mirrors find-ings in other reports where this angle does not correlate with the magnitude of calcaneal valgus.In our patients with flat Foot, whose main pathology is in midtarsal, show substantial clinical valgus with only slightly increased talocaicaneal angle. I our study we noted that this angle decreases normally during aging.

Tagehi et al were doing a calcaneal lengthening osteotomy was performed in 20 patients. 30 feet, with idiopathic flexible flatfoot that was failed to conservative treatment between 2007 and 2011. Patients were evaluated by ACFAS universal evaluation scoring scale and radiographic indexes. The mean follow up duration was $23.1 \pm 9.9$ months the average age was $104 \pm 0.9$ years. Achilles tendon lengthening was done in 28 feet. ACFAS score at the final follow up had increased significantly compared to pre-operative score (37 to 88, $\mathrm{P}<0.0001)$. Radiographic parameters also showed significant improvement after surgery $((\mathrm{P}<0.0001))$. Distal segment displacement and hardware irritation as postop complications were found in 2 and 3 cases, respectively, with no long-term clinical impact. (23)

\section{CONClusion}

In our study can be done safely in young children and adolescent as there is no interference with the centers of ossification of the foot bones. K-wire fixation instead of screws or Steinmann pins gave the advantage of being cheaper, easier to apply and avoids the need of a second step for removal. A long term follow up is advised to prove the maintenance of correction and absence of recurrence.

Finally, there was excellent agreement between clinical, pedography and radiological results.

\section{RECOMMENDATION}

Soft tissue and bony procedures used to recon struct (FFF) can also be safely applied to adolescents and young adults. Both radiological and clinical improvements were observed in present study group.

\section{REFERENCES}

[1] Gray's Anatomy,By SusanStandring; 39th Edition; chapter 115-Foot and ankle; 1507:45.107 (2005)

[2] Mosca vs. : The child's .foot.: principles b.f management [J Pediatr Ortbop.18:281282.(1998)

[3] Tylkowski,C.M. (1990): Dynamics of the foot in gait. In Lovell \& Winter pediatric orthopedics Lippincott Philadelphia. .PP.7 I 
[4] Vincent S. Mosca: Flexible flatfoot in children and adolescents. J Child Orthop 4:107-12. (2010)

[5] Williams P. And Dyson M.. : Lower limb. Gray's anatomy, Churchill Livingstone ,UK, 38th edition: 7 12-736 (1995)

[6] Anand M. Vop MD, Tudor R. Tien, MD, Brent G. Parks, MSC, and Lew C. Schon, MD: Correction of Moderate and Sever Acqurated Flexible Flatfoot with Medialization Calcanel Osteotomy and Flexor Digltorum Longus Transfer the Journal of Bone and Joint Surgery, (1726-1734) Volume 88-A . Number 8 August (2006).

[7] Alessio Bernasconi' François Lintz Francesco Sadile: The role of arthroereisis of the subtalar joint for flatfoot in children and adults. Volume 2 , November (2017)

[8] 8-Bendetti G and Berti L: clinicoradiographic assessment of flexible flat foot in children. $G$ AM paid-Med. Assoc. 20/10November .100(6) $(463-71)$.

[9] Jeffrey E. Johnson MD, BrUCe Cohen MD, Benedict F. DiGiovanni MD, Ron Lamdan MD St. Louis, Mo; Tallahassee, FL; Rochester, NY and Milwaukee. WI : Subtalar Arthrodesis with Flexor Digitorum Longus Transfer and Spring Ligament Repair for Treatment of Posterior Tibial Tendon Insufficiency. Foot \& Ankle IntarnationalNol. 21, No. 9/September (722729). (2000)

[10] Kent R. Di Nucci, DPM, Jeffrey C: Biomechanical Consequences of Lateral Column Lengthening of the Calcaneus: Part I. Long Plantar Ligament Strain The Journal of Foot \& Ankle Surgery, 43(1):10 — 15. (2004)

[11] Rodriguez N, Volpe RG: Clinical diagnosis and assessment of the pediatric pes planovalgus deformity. Clin Podiatr Med Surg. Jan; 27(1):43-58 (2010)

[12] Blitz NM. Pediatric: Adolescent flatfoot reconstruction in combination middle facet talocalcaneal coalition resection. Clin Podiatr Med Surg. ; 27:119-133. (2010)

[13] Mickle KJ, Steele JR, Munro BJ : The feet of overweight andobese young children: are they flat or fat? Obesity (Silver Spring) 14:19491953 (2006)
[14] Evans AM, Rome K. : A Cochrane review of the evidence for non- surgical interventions for flexible pediatric flat feet. Eur J Phys Rehab Med. 47(1):69—89. (2011)

[15] Mosca VS. Flexible flatfoot in children and adolescents. Child Orthop. 2010; 4(2):107-21.

[16] Martinus Richter MD, PhD Stefan Zech MD: Lengthening osteotomy of the calcaneus and flexor digitorum longus tendon transfer in flexible flatfoot deformity improves talo-Ist metatarsal-Index, clinical outcome and pedographic parameter Yon tar et at. / Acta Orthopoedica et Traumatotogica Turcica 50 655-659(2016)

[17] Pfeiffer M, Katz R, Ledi T, Hauser G, Sluga M. Prevalence of flat foot in preschool aged children. Pediatrics. 2006; 118(2):634-9.

[18] Phillips GE. A review of elongation of os calcis for flat feet. J Bone Joint Surg Br. 1983; 65(1):15-8.

[19] Mosca VS. Calcaneal lengthening for valgus deformity of the hindfoot. Results in children who had severe, symptomatic flatfoot and skewfoot. J Bone joint Surg Am. 1995; 77(41)

[20] Dogan A, Albayrak M, Akman E, Zorer G. The result of calcaneal lengthening ostetomy for the treatment of flexible pes planovalgus and evaluation of alignment of the foot. Acta Orthop Traumatol Turc. 2006; 40 (5):3 56-66.

[21] Viegas $G$ Re struc of pediatric flexible planovalgus foot by using $r$ calcaneal osteotomy and augmentative medial split tibi uerior i transfer. 1 Foot Ankle Surg. 2003; 42(4): (19)

[22] Reilingh ML, Beimers L, Tuijthof GJ, Stufkens SA, Maas $M$, van Dijk CN. Measur-ing hindfoot alignment radiographically: the long axial view is more reliable than the hindfoot alignment view. Skeletal Radiol.2010; 39(11):1103-8.

[23] (Taghi Baghdadi, MD; Hamed Mazoochy, MD; Mohammadreza Guity, MD; Nima 1-leidari khabbaz, MD) Evaluation of Clinical and Radiological Results of Calcaneal Lengthening Osteotomy in Pediatric Idiopathic Flexible Flatfoot Arch Bone JtSurg. 2018; 6(5):402-411.

[24] Evans D. Calcaneo-valgus deformity. j Bone Joint Surg Br. 1975; 57(3):270-8.

Citation: Mohamed Abd-El Aziz Mohamed Ali, Mohammed Mansour, Nagy Fouda. Combined Lateral Calcaneal Lengthening Osteotomy and Dynamic Soft Tissue Reconstruction of Medial Foot in Adolescent Flexible Flatfoot. ARC Journal of Orthopedics. 2020; 5(1):21-31. doi:dx.doi.org/10.20431/24560588.0501004.

Copyright: (c) 2020 Authors. This is an open-access article distributed under the terms of the Creative Commons Attribution License, which permits unrestricted use, distribution, and reproduction in any medium, provided the original author and source are credited. 